\title{
A simple grid implementation with Berkeley Open Infrastructure for Network Computing using BLAST as a model
}

Watthanai Pinthong, Panya Muangruen, Prapat Suriyaphol, Dumrong Mairiang

Development of high-throughput technologies, such as Next-generation sequencing, allows thousands of experiments to be performed simultaneously while reducing resource requirement. Consequently, a massive amount of experiment data is now rapidly generated. Nevertheless, the data are not readily usable or meaningful until they are further analysed and interpreted. Due to the size of the data, a high performance computer (HPC) is required for the analysis and interpretation. However, the HPC is expensive and difficult to access. Other means were developed to allow researchers to acquire the power of HPC without a need to purchase and maintain one such as cloud computing services and grid computing system. In this study, we implemented grid computing in a computer training center environment using Berkeley Open Infrastructure for Network Computing (BOINC) as a job distributor and data manager combining all desktop computers to virtualize the HPC. Fifty desktop computers were used for setting up a grid system during the off-hours. In order to test the performance of the grid system, we adapted the Basic Local Alignment Search Tools (BLAST) to the BOINC system. Sequencing results from Illumina platform were aligned to the human genome database by BLAST on the grid system. The result and processing time were compared to those from a single desktop computer and HPC. The estimated durations of BLAST analysis for 4 million sequence reads on a desktop PC, HPC and the grid system were 568, 24 and 5 days, respectively. Thus, the grid implementation of BLAST by BOINC is an efficient alternative to the HPC for sequence alignment. The grid implementation by BOINC also helped tap unused computing resources during the off-hours and could be easily modified for other available bioinformatics software. 


\section{A Simple Grid Implementation with Berkeley Open Infrastructure}

2 for Network Computing using BLAST as a Model

3

4 Watthanai Pinthonga ${ }^{\mathrm{a}}$, Panya Muangruen ${ }^{\mathrm{b}}$, Prapat Suriyaphol $\mathrm{l}^{\mathrm{c}, *}$, Dumrong Mairiangd,e,**

5 a Department of Anatomy, Faculty of Medicine Siriraj Hospital, Mahidol University, Bangkok

610700 Thailand

7 biriraj Information Technology Department, Faculty of Medicine Siriraj Hospital, Mahidol

8 University, Bangkok 10700 Thailand

$9{ }^{c}$ Division of Bioinformatics and Data Management for Research, Department of Research and

10 Development, Faculty of Medicine Siriraj Hospital, Mahidol University, Bangkok 10700,

11 Thailand

12

${ }^{d}$ Medical Biotechnology Research Laboratory, The National Center for Genetic Engineering and Biotechnology, National Science and Technology Development Agency, Pathumthani 11000, Thailand

${ }^{e}$ Division of Dengue Hemorrhagic Fever Research, Department of Research and Development, Faculty of Medicine Siriraj Hospital, Mahidol University, Bangkok 10700, Thailand

\section{ABSTRACT}

Development of high-throughput technologies, such as Next-generation sequencing, allows thousands of experiments to be performed simultaneously while reducing resource requirement. Consequently, a massive amount of experiment data is now rapidly generated. Nevertheless, the data are not readily usable or meaningful until they are further analysed and interpreted. Due to the size of the data, a high performance computer (HPC) is required for the analysis and interpretation. However, the HPC is expensive and difficult to access. Other means were developed to allow researchers to acquire the power of HPC without a need to purchase and maintain one such as cloud computing services and grid computing system. In this study, we implemented grid computing in a computer training center environment using Berkeley Open Infrastructure for Network Computing (BOINC) as a job distributor and data manager combining all desktop computers to virtualize the HPC. Fifty desktop computers were used for setting up a grid system

\footnotetext{
*Corresponding author. E-mail: dumrong.mai@biotec.or.th, prapat.sur@mahidol.ac.th
} 
29 during the off-hours. In order to test the performance of the grid system, we adapted the Basic 30 Local Alignment Search Tools (BLAST) to the BOINC system. Sequencing results from Illumina 31 platform were aligned to the human genome database by BLAST on the grid system. The result 32 and processing time were compared to those from a single desktop computer and HPC. The 33 estimated durations of BLAST analysis for 4 million sequence reads on a desktop PC, HPC and 34 the grid system were 568, 24 and 5 days, respectively. Thus, the grid implementation of BLAST 35 by BOINC is an efficient alternative to the HPC for sequence alignment. The grid implementation 36 by BOINC also helped tap unused computing resources during the off-hours and could be easily 37 modified for other available bioinformatics software.

\section{INTRODUCTION}

Massive data are now affordably, easily and frequently generated by genomic and proteomic assays such as massively parallel sequencing and high-throughput mass spectrometry. Up to 1 trillion bases can be sequenced in one 6-day run by Illumina HiSeq 2500 (Rhoads \& Au 2015) while mass spectrometry can now completely analyse a proteome and quantify protein concentrations in an entire organism (Ahrne et al. 2015). Breakthroughs in genomic and proteomic data generation lead to development and emergence of several disciplines. In precision medicine, clinicians can diagnose and tailor a treatment for a disease based on the patient profile derived from "omics" data (Chen \& Snyder 2012). Furthermore, metagenomics, a study of genetic materials in samples directly collected from particular environments, is now greatly advanced by high-throughput assays, and now becomes applicable to forensic sciences (Fierer et al. 2010) and pathogen discovery (Chiu 2013). However, genomic and proteomic data are not readily usable or meaningful without proper analysis and interpretation which become the bottleneck of genomic and proteomic studies due to tremendous computational resource requirement (Scholz et al. 2012) (Berger et al. 2013) (Neuhauser et al. 2013).

To overcome the bottleneck of data analysis, high performance computing (HPC) is now commonly used in large-scale bioinformatics tasks including sequence alignment (Orobitg et al. 2015), simulation (Zhang et al. 2014) and machine learning (D'Angelo \& Rampone 2014). The physical architecture of HPC consists of numerous processing units, large shared memory and huge data storage cooperatively functioning to obtain high performance usually measured as floating-point operations per second (FLOPS) (Subramaniam \& Feng 2012). However, physical 
60 HPC is costly and requires extensive maintenance. Cloud computing services, such as Amazon

61 EC2, is now an alternative to purchase a physical HPC for scientific computing (Juve et al.

62 2009). Several bioinformatics applications and frameworks are now designed to utilize cloud

63 computing and/or grid computing such as CloVR (Angiuoli et al. 2011a), Galaxy (Blankenberg

64 et al. 2010), Tavaxy (Abouelhoda et al. 2012) and CloudBurst (Schatz 2009). Bioinformatics

65

66

67

68

69

70

71

72

73

74

75

76

77

78

79

80

81

82

83

84

85

86

87

88

89

90 analyses usually involve repetitive computing intensive tasks that can be split into several smaller and less computing intensive tasks (Carvalho et al. 2005). Thus, massive parallelization on HPC or cloud computing was usually employed for large-scale bioinformatics analyses.

MapReduce framework, such as Apache Hadoop (White 2012), is usually employed for massive parallelization in which input data are split and mapped to worker nodes while output data from worker nodes were merged or reduced at the head node (Dean \& Ghemawat 2008). Recently, Apache Spark has become another framework for parallelization and cluster computing (Zaharia et al. 2010). Nevertheless, both physical HPC and cloud computing are still too costly for some research groups and require personnel with advanced computing skills to manage the systems.

There are also large public grid systems such as Open Science Grid (Pordes et al. 2007) that were used for bioinformatics data analysis including BLAST analysis (Hayashi et al. 2014). However, large inter-institutional grid systems may not always be suitable under certain circumstances. For example, limited bandwidth and firewalls can hinder the data transfer to and from remotely located grid systems. In addition, clinical data must be carefully protected so transferring them to the public grid system may risk privacy violation. Also, if a grid system requires a dedicated server with routine maintenance, it will not be affordable for a small research group. A local grid system, which could be easily assembled, disassembled and then reassembled, will benefit a research group that could intermittently access abundant, but not individually powerful, computing resources such as a computer training center.

Berkeley Open Infrastructure for Network Computing (BOINC) is a middleware that helps manage volunteer and grid computing (Anderson 2004). One of the best known BOINCbased projects is SETI@home whose purpose is to search for signs of extra terrestrial intelligence from radio telescope data (Anderson et al.2002). SETI@home project recruits home computers to help analyse small chunks of data during the idle time. Data are sent and received through the internet creating a large distributed computing system. BOINC also supports an implementation of grid computing on local desktop computers (Balaton et al. 2007). Academic 
91 and research institutes usually own several desktop computers, which are idle during the off-

92 hours. These unused computer resources can be tapped by implementing grid computing with

93 BOINC. The BOINC-based grid computing could inexpensively provide adequate computing

94 power required by many bioinformatics analyses.

95 Sequence alignment is one of the basic analyses for genomic and proteomic data and

96 arranges DNA, RNA or protein sequences against one another or sequence databases to detect

97 similarities or differences in order to infer functional, structural, or evolutionary relationships

98 (Baxevanis \& Ouellette 2001). Basic Local Alignment Search Tools (BLAST) (Altschul et al.

99 1990) is a program widely used for sequence alignment. However, BLAST is not optimized for

100 analysing massive data generated by high-throughput assays, and using BLAST for high-

101 throughput data may lead to impractical runtimes (Li et al. 2008) (Borozan et al. 2013). Thus,

102 other sequence alignment programs have been developed such as BWA (Li \& Durbin 2009) and

103 Bowtie (Langmead et al. 2009) to handle short sequences generated by high-throughput assays.

104 As these methods are mostly specialized to only handle short sequences, several methods have

105 been applied to improve the performance of BLAST in order to handle large data such as using

106 parallel processing (Darling et al. 2003), grid computing (Carvalho et al. 2005) and cloud 107 computing (Angiuoli et al. 2011b).

This study did not, however, aim to develop a novel method to increase the speed of

109

110

111

112

113

114

115

116

117

118

119

120

121

sequence alignment. We aimed to use BLAST as a model for grid implementation because it is one of the most commonly used bioinformatics tools (Carvalho et al. 2005) with good documentation. Furthermore, BLAST can be performed in parallel without the need to communicate among worker nodes during processing (Mathog 2003). There was a study by Pellicer et al., that had applied BOINC to BLAST to increase the alignment speed (Pellicer et al. 2008). Our study aimed to confirm that the grid implementation with BOINC proposed by Pellicer et al., functioned well with actual next-generation sequencing data. In addition, we aimed to document instructions on how to set up BOINC grid system. The BLAST model for grid computing using BOINC is the first step to evaluate and design a simple grid

implementation, which will help research groups with limited computing resources tap into idle computers in their organizations for large-scale bioinformatics analyses.

\section{MATERIALS AND METHODS}

\section{Sequences and Databases}


122

To test sequence alignment with BLAST, human DNA sequencing data (genome ID:

123 NA12878) was downloaded from the Genome in the Bottle Consortium (ftp://ftp-

124 trace.ncbi.nlm.nih.gov/giab/ftp/data/NA12878/NIST_NA12878_HG001_HiSeq_300x) (Zook et

125 al. 2014). NA12878 data were from Illumina HiSeq2500 sequencing platform with about 300x

126 total coverage of $150 \times 150 \mathrm{bp}$. The data contained 4 million sequence reads in fastq format.

127 However, fastq format was not compatible with the BLAST program so the data were converted

128 to fasta format using seqIO command of Biopython (version 1.65) (Cock et al.

129 2009)(https://github.com/dummai/BoincBlastTest/blob/master/PyScripts/convertFastqToFaQual.

130 py). Since it would take an impractical amount of time to perform BLAST analysis on all 4

131 million sequence reads, the data were split into smaller chunks including read 1 only, read 1 to

13210 , read 1 to 100 , read 1 to 1,000 , read 1 to 10,000 and read 1 to 100,000 . For task distribution

133 by BOINC, the whole data were split into 40,000 smaller files each containing 100 reads with

134 Chunk 1 containing read 1 to read 100, Chunk 2 containing read 101 to read 200 and so forth

135 using a Python script

136 (https://github.com/biopython/biopython.github.io/blob/master/wiki/Split_large_file.md).

137 NA12878 data were aligned to the human genome, Genome Reference Consortium Human

138 Reference 38 (hg38), downloaded from UCSC Genome Bioinformatics

139 (http://hgdownload.cse.ucsc.edu/goldenPath/hg38/bigZips/) (Rosenbloom et al. 2015). The

140 human genome (hg38) were formatted with the makeblastdb command of BLAST program.

141 BLAST Analysis

142

NCBI BLAST+ (version 2.2.30) for Microsoft ${ }^{\circledR}$ Windows 32-bit and for Linux/GNU 32-

143 bit were downloaded from the National Center for Biotechnology Information repository

144 (ftp://ftp.ncbi.nlm.nih.gov/blast/executables/blast+/2.2.30/) (Camacho et al. 2009). blastn

145 command of NCBI BLAST+ was used to align NA12878 sequences against hg38. The output

146 format was set to tabular with comment lines (-outfmt 7) and filtering with dust was turned off (-

147 dust no). Finally, any hit that was enveloped by at least 20 higher-scoring hits was deleted (-

148 culling_limit 20). The same blastn command line with the aforementioned settings was used for

149 sequence alignment on all platforms. Read 1 only, read 1 to 10 , read 1 to 100 and read 1 to 1,000

150 were used for BLAST analysis on a desktop computer. Read 1 to 10,000 and read 1 to 100,000

151 were additionally used for BLAST analysis on a HPC. All sequence alignments, except for read

1521 to 100,000 set on HPC, were conducted in triplicate to derive an average processing time. 
153 Computers for BLAST analysis

154

155

156

157

158

159

160

161

162

163

164

165

166

167

168

169

170

171

172

173

174

175

176

177

178

179

180

181

182

183

The specification of the computer used for BLAST analysis was Intel ${ }^{\circledR}$ Core $^{\mathrm{TM}} \mathrm{i} 7-4500 \mathrm{U}$ CPU@2.40 GHz with 8.00 GB of RAM. To simulate resources used per one workunit on BOINC grid system, hardware virtualization was required to allocate one processing unit and $1.00 \mathrm{~GB}$ of RAM. The virtualization was done by using Oracle ${ }^{\circledR}$ VM VirtualBox (version 5.0.8). The operating system for virtualization was Microsoft@ Windows 7 (32-bit).

\section{HPC for BLAST analysis}

The specification of the HPC used for BLAST analysis was Cisco® UCS Blade Server B200M2 $\times 2$ Units with CPU $2 \times 6$ cores $(2.4 \mathrm{GHz}, 12$ cores in total) and 96 GB of RAM. The HPC could use up to 24 processing threads simultaneously. The operating system of the HPC was Ubuntu Server Linux/GNU (version 14.04 TLS). BLAST analysis was restricted to run on 12 processing threads by adding '-num_threads 12' to the blastn command line.

\section{Computer training center for grid implementation}

A computer training room with 50 desktop computers was used for grid system implementation. The room was accessible from 5PM to 8AM, after its regular operating hours. The specification of each computer was Intel® Core ${ }^{\mathrm{TM}}$ i5 CPU $660 @ 3.47$ GHz with 4.00 GB of RAM. The operating system of all computers was Microsoft ${ }^{\circledR}$ Windows 7 (32-bit). Every computer was connected to local area network. One computer was assigned as a host machine for the BOINC project server. This computer had Oracle ${ }^{\circledR}$ VM VirtualBox (version 5.0.8) installed to virtualize hardware for the project server. The virtual machine image of the BOINC project server (version April 12, 2014 on Debian Linux/GNU version 7) was downloaded from BOINC webpage (https://boinc.berkeley.edu/dl/debian-7-boinc-server-140412.7z). One processing unit and $1.00 \mathrm{~GB}$ of RAM were allocated for the project server. Other computers were assigned as client machines with BOINCManager (version 7.4.42;

https://boinc.berkeley.edu/dl/boinc_7.4.42_windows_intelx86.exe) installed.

\section{Grid Implementation by BOINC}

Documentation for grid implementation by BOINC are available at https://github.com/dummai/BoincBlastTest. BLAST program must be adapted to BOINC system for distributed computing. Thus, BOINC wrapper program (version 26014 for Microsoft ${ }^{\circledR}$ Windows 32-bit; https://boinc.berkeley.edu/dl/wrapper_26014_windows_intelx86.zip) along with an XML script (https://github.com/dummai/BoincBlastTest/tree/master/xml) were applied 
184 to the batach file containing a command line of blastn program and time recording commands 185 (https://github.com/dummai/BoincBlastTest/blob/master/bat/blastn_windows_intelx86_0.bat). 186 An XML script was used as a template for how to send clients input data including the hg38

187 database and each chunk of sequencing reads

188 (https://github.com/dummai/BoincBlastTest/blob/master/xml/blastn_wu). Another XML script 189 was used as a template for how to return BLAST analysis result back from the clients

190 (https://github.com/dummai/BoincBlastTest/blob/master/xml/blastn_re). Python scripts were 191 used to create bash shell scripts for staging input files

192 (https://github.com/dummai/BoincBlastTest/blob/master/PyScripts/stage_file.py) and creating 193 workunits (WUs;

194 https://github.com/dummai/BoincBlastTest/blob/master/PyScripts/create_wu.py). One WU is a 195 job package containing input data, application and instruction to be sent and processed at the 196 client machines. Since this is a grid computing system, an option to create redundant WUs for 197 results verification required in BOINC volunteer system was turned off. Due to limited time 198 allocation for accessing the training center, only 5,000 files containing 500,000 sequence reads 199 were tested during one overnight run. The overall processing time was calculated from the 200 difference between the time the first WU was sent and the time the last result received. In 201 addition, the time points at which each WU was sent out, started processing, finished processing 202 and retrieved back were recorded. Since a lagging process on a single machine could delay the 203 overall processing time, a time limit of one hour was set to terminate a process taking longer than 204 one hour and resend to other machine. To ensure that the grid implementation did not affect the 205 results of BLAST analysis, the sequence alignment results of read 1 to 1,000 from the desktop 206 computer and BOINC grid were compared.

207

208 RESULTS AND DISCUSSION

\section{Sequence alignment without grid implementation}

Sequence alignment with BLAST program could be performed on a web-based application at NCBI webpage (http://blast.ncbi.nlm.nih.gov/Blast.cgi). However, this is not a 212 suitable option for analysing a large number of query sequences due to its dependence on

213 network connection for query submission and result download. In addition, NCBI does not 214 provide computer resources for all large-scale analyses submitted through the web-based 
215 application. Thus, standalone BLAST is provided by NCBI for running sequence alignment 216 locally on a computer or HPC. In this study, standalone BLAST was chosen to benchmark 217 analysis time of a desktop computer and HPC against that of BOINC grid system.

218 From a preliminary run of BOINC grid system, we found that one client machine could 219 process up to four WUs simultaneously (data not shown). This was later confirmed when full 220 grid implementation by BOINC was tested (Figure 5). To benchmark the processing time of a 221 single computer against BOINC grid system, we restricted computing resources for running 222 standalone BLAST on the computer by hardware virtualization to the same resources used for 223 processing one WU in BOINC grid system, which included one central processing unit and 1.00 224 GB of RAM. Additionally, the computing resources restriction helped estimate the time required 225 for processing one WU with various number of sequence reads on BOINC grid system. Thus, an 226 optimal number of sequence reads per WU could be selected. One, ten, 100 and 1,000 sequence

227 reads were aligned against the human genome (figure 1). The average processing time for 228 sequence alignment of 1,000 reads was 204.17 minutes which was too long for processing one 229 WU. An error at the end of processing a WU with 1,000 reads would result in a loss of three and 230 a half hours of processing time. Thus, WU's with 100 reads was used for analysis on BOINC 231 grid, which would take approximately 20 minutes per WU. Simple linear regression analysis was 232 used to predicted the analysis time for all 4 million reads. Based on an estimated 12.28 seconds 233 to analyse a single read, it would take approximately 568 days to complete the sequence 234 alignment with a linear approach. However, multiprocessing and multithreading capabilities of 235 the current personal computer would significantly reduce the overall processing time depending 236 on its central processing unit and shared memory.

237 Currently, HPC is used in several bioinformatics analyses (D'Angelo \& Rampone 2014;

238 Orobitg et al. 2015; Zhang et al. 2014). HPC can manage large datasets and handle intensive 239 computation while significantly reduce the processing time. However, HPC is expensive and 240 requires extensive maintenance. In this study, HPC was used for benchmarking the time used for 241 sequence alignment against that of the alignment on BOINC grid. Twelve threads of HPC were 242 simultaneously used for BLAST program. From the initial dataset of 4 million reads, one, ten, $243100,1,000,10,000$ and 100,000 sequence reads were aligned against the human genome (figure 244 2). The sequence alignment of 100,000 reads took about 880 minutes. Simple linear regression 245 analysis was used to predict the relationship between number of reads and total analysis time on 
246 the HPC. An estimated 0.53 seconds per read meant it would take approximately 24 days to 247 complete the sequence alignment for all 4 million reads. The HPC used in the study was a small 248 model, however, a larger HPC might be able to complete the same sequence alignment within a 249 few days.

\section{Sequence alignment with grid implementation by BOINC}

BOINC helps manage distribution of a large number of tasks to client machines on

252

253

254

255

256

257

258

259

260

261

262

263

264

265

266

267

268

269

270

271

272

273

274

275

276

volunteer or grid system. In addition, BOINC has a system to validate the results returned by the clients. However, the BOINC project administrator is required to prepare input data for task distribution as well as plan methods to consolidate result files into a single final result. In this study, 4 million sequence reads were split into 40,000 files with each file containing 100 sequence reads. The number of files was decided to minimize the number of files while keeping total runtime at a reasonable level (figure 1). The human genome database was also sent to client machines along with a sequence file. However, a "sticky" option of BOINC ensured that the database was transferred to the same client only once and remained in the machine for other rounds of sequence alignment.

In this study, BOINC grid system was tested in one overnight session. One session was expected to be sufficient for sequence alignment of 500,000 reads. Consequently, Chunk 1 to Chunk 5,000 of data, which contained read 1 to read 500,000 were used during this session. The time between when the first file was sent to the client and the time the last result file was received by the project server was 846 minutes or about 14 hours. The cumulative elapsed time from each WU was 90,400 minutes meaning the grid system reduced the real processing time by a factor of 107 . The average processing time of the WUs was 18 minutes with a range of 5 to 48 minutes (figure 3). The client machines showed a bimodal distribution in running time, with one group having an average processing time per WU at about 16 to 18 minutes while a minority group had a longer processing time per WU at more than 23 minutes (Figure 4A). To measure whether data transfer speed via the local area network significantly affected the processing time at each client machines, the runtime of each blast analysis were also recorded at the client machines. The difference between the processing time recorded at the server and that at the client machine was the time used for data transfer. The average data transfer time was 41 seconds with a range of 16 to 219 seconds (Figure 4B). The delay caused by data transfer speed was small compared to the actual times used for BLAST analysis. Thus, data transfer speed did not affect 
277 the processing time observed in the client machines. It was impossible to directly measure the

278 times used for sending or receiving data since the clocks of the project server and client

279 machines were not synchronized. During the overnight session, an average number of WUs

280 processed by each client was 104 with a range of 92 to 140 WUs (Figure 4C) resulting in an

281 average of 10,400 processed reads. A minority of client machines had processed 130 WUs or

282 more (Figure 4C). Interestingly, the minority of client machines with longer average processing

283 times and the client machines with more WUs processed were exactly the same machines. When

284 the number of WUs being processed at a particular time was tracked, we found that the slow

285 group simultaneously processed 4 WUs most of the time while the fast groups only

286 simultaneously processed 2 WUs most of the time. The machine with the longest average

287 processing time and the one with the shortest processing time were selected for tracking the

288 number of WUs being processed in Figure 5. We suspected that the configuration of hardware

289 (other than CPU and RAM) and/or software (background application) of these two groups were

290 different. BOINC grid system is designed to cope with clients with heterogeneous configurations

291 and specifications so the difference in the ability to process WUs in parallel would not cause a

292 major problem.

293 The sequence alignment of read 1 to 1,000 from BOINC grid

294 (https://github.com/dummai/BoincBlastTest/blob/master/raw/BOINC 1000reads_BLASTresults.

295 txt) were compared to the results from the single computer

296 (https://github.com/dummai/BoincBlastTest/blob/master/raw/singlePC_1000reads_BLASTresuls

297 .txt). The results were identical. Thus, the BOINC grid system helped increase the speed of

298 sequence alignment greatly and did not affect the final results. Using the same BOINC grid

299 setup, it would take 8 overnight sessions or one 5-day-long session to finish the sequence

300 alignment of 4 million reads. The BOINC grid is highly scalable and when more clients join the

301 grid the overall processing speed of the grid would proportionally increase.

302 There are other middleware or systems for grid implementation including Condor

303 (Epema et al. 1996), Univa Grid Engine (http://www.univa.com/products/) and PBS/Torque

304 (http://www.adaptivecomputing.com/products/open-source/torque/). We did not select Univa

305 Grid Engine because it is proprietary. PBS/Torque system is not compatible with a computer

306 training center with machines running on Microsoft Windows. Condor, now known as

307 HTCondor, is a comparable alternative to BOINC for grid implementation in a computer training 
308 center environment. Condor has an advantage over BOINC as the modification of an application

309 to be distributed is not required if the application has binary compatibility (Søttrup \& Pedersen

310 2005). However, Condor is not suitable with volunteer computing that requires validation

311 strategy whereas BOINC utilizes homogenous redundancy to do so (Søttrup \& Pedersen 2005).

312 In this study, BOINC was selected because there were more projects similar to this study

313 available as reference and instructions of the initial installation and configuration were available.

314 BOINC grid system still has some limitations. In metagenomics analyses, sequencing

315 data might be aligned against the nucleotide collection database ( $\mathrm{nr}$ database). The nr nucleotide

316 database is large and it would be difficult to distribute the database to each client. A strategy for

317 splitting nr database and merging alignment results would be necessary. BOINC grid does not

318 allow clients to directly contact one another. Thus, some applications that require client

319 communication would not be suitable for grid implementation with BOINC. On the other hand,

320 the BOINC grid system allows clients with various operating systems as long as they are

321 compatible to the application used in the grid system. The wrapper program for BOINC provides

322 convenient methods to adapt applications for grid implementation.

323 We created a series of documentation for setting up of BOINC grid system to help other

324 research groups with limited computing resources tap unused computer in their institutes

325 (https://github.com/dummai/BoincBlastTest).

\section{CONCLUSIONS}

Many academic and research institutes have under-utilized computing resources in the

form of computer training centers. In this study, we showed a way to potentially tap these unused resources by grid implementation with BOINC. We tested the grid system using BLAST analysis as a model. The results showed that the grid system greatly increase the speed of BLAST analysis without affecting the quality of the results. Thus, the grid implementation with BOINC would be an economically alternative to HPC for any research groups with limited resources.

\section{ACKNOWLEDGMENTS}

We would like to thank Associate Professor Thawornchai Limjindaporn for his valuable advice on this project and Dr. Harald Grove for very helpful English editing. 


\section{REFERENCES}

338

339

340

341

342

343

344

345

346

347

348

349

350

351

352

353

354

355

356

357

358

359

360

361

362

363

364

365

366

367

368

369

370

371

372

373

374

375

376

377

378

379

380

Abouelhoda M, Issa SA, and Ghanem M. 2012. Tavaxy: integrating Taverna and Galaxy workflows with cloud computing support. BMC Bioinformatics 13:77. 10.1186/14712105-13-77

Ahrne E, Martinez-Segura A, Syed AP, Vina-Vilaseca A, Gruber AJ, Marguerat S, and Schmidt A. 2015. Exploiting the multiplexing capabilities of tandem mass tags for highthroughput estimation of cellular protein abundances by mass spectrometry. Methods 85:100-107. 10.1016/j.ymeth.2015.04.032

Altschul SF, Gish W, Miller W, Myers EW, and Lipman DJ. 1990. Basic local alignment search tool. J Mol Biol 215:403-410. 10.1016/S0022-2836(05)80360-2

Anderson DP. 2004. Boinc: A system for public-resource computing and storage. Grid Computing, 2004 Proceedings Fifth IEEE/ACM International Workshop on: IEEE. p 410.

Anderson DP, Cobb J, Korpela E, Lebofsky M, and Werthimer D. 2002. SETI@ home: an experiment in public-resource computing. Communications of the ACM 45:56-61.

Angiuoli SV, Matalka M, Gussman A, Galens K, Vangala M, Riley DR, Arze C, White JR, White O, and Fricke WF. 2011a. CloVR: a virtual machine for automated and portable sequence analysis from the desktop using cloud computing. BMC Bioinformatics 12:356. 10.1186/1471-2105-12-356

Angiuoli SV, White JR, Matalka M, White O, and Fricke WF. 2011b. Resources and costs for microbial sequence analysis evaluated using virtual machines and cloud computing. PLoS One 6:e26624. 10.1371/journal.pone.0026624

Balaton Z, Gombás G, Kacsuk P, Kornafeld A, Kovács J, Marosi AC, Vida G, Podhorszki N, and Kiss T. 2007. Sztaki desktop grid: a modular and scalable way of building large computing grids. Parallel and Distributed Processing Symposium, 2007 IPDPS 2007 IEEE International: IEEE. $\mathrm{p} \mathrm{1-8.}$

Baxevanis AD, and Ouellette BFF. 2001. Bioinformatics : a practical guide to the analysis of genes and proteins. Methods of biochemical analysis v 43. 2nd ed. New York: WileyInterscience. $p$ xviii, 470 p., 413 p. of plates.

Berger B, Peng J, and Singh M. 2013. Computational solutions for omics data. Nat Rev Genet 14:333-346. $10.1038 / \mathrm{nrg} 3433$

Blankenberg D, Von Kuster G, Coraor N, Ananda G, Lazarus R, Mangan M, Nekrutenko A, and Taylor J. 2010. Galaxy: a web-based genome analysis tool for experimentalists. Curr Protoc Mol Biol Chapter 19:Unit 19 10 11-21. 10.1002/0471142727.mb1910s89

Borozan I, Watt SN, and Ferretti V. 2013. Evaluation of alignment algorithms for discovery and identification of pathogens using RNA-Seq. PLoS One 8:e76935. 10.1371/journal.pone.0076935

Camacho C, Coulouris G, Avagyan V, Ma N, Papadopoulos J, Bealer K, and Madden TL. 2009. BLAST+: architecture and applications. BMC Bioinformatics 10:421. 10.1186/14712105-10-421

Carvalho PC, Gloria RV, de Miranda AB, and Degrave WM. 2005. Squid - a simple bioinformatics grid. BMC Bioinformatics 6:197. 10.1186/1471-2105-6-197

Chen R, and Snyder M. 2012. Systems biology: personalized medicine for the future? Curr Opin Pharmacol 12:623-628. 10.1016/j.coph.2012.07.011 
381

382

383

384

385

386

387

388

389

390

391

392

393

394

395

396

397

398

399

400

401

402

403

404

405

406

407

408

409

410

411

412

413

414

415

416

417

418

419

420

421

422

423

424

425

426

Chiu CY. 2013. Viral pathogen discovery. Curr Opin Microbiol 16:468-478. 10.1016/j.mib.2013.05.001

Cock PJ, Antao T, Chang JT, Chapman BA, Cox CJ, Dalke A, Friedberg I, Hamelryck T, Kauff F, Wilczynski B, and de Hoon MJ. 2009. Biopython: freely available Python tools for computational molecular biology and bioinformatics. Bioinformatics 25:1422-1423. 10.1093/bioinformatics/btp163

D'Angelo G, and Rampone S. 2014. Towards a HPC-oriented parallel implementation of a learning algorithm for bioinformatics applications. BMC Bioinformatics 15 Suppl 5:S2. 10.1186/1471-2105-15-S5-S2

Darling A, Carey L, and Feng W-c. 2003. The design, implementation, and evaluation of mpiBLAST. Proceedings of ClusterWorld 2003:13-15.

Dean J, and Ghemawat S. 2008. MapReduce: simplified data processing on large clusters. Communications of the ACM 51:107-113.

Epema DHJ, Livny M, van Dantzig R, Evers X, and Pruyne J. 1996. A worldwide flock of Condors: Load sharing among workstation clusters. Future Generation Computer Systems 12:53-65. http://dx.doi.org/10.1016/0167-739X(95)00035-Q

Fierer N, Lauber CL, Zhou N, McDonald D, Costello EK, and Knight R. 2010. Forensic identification using skin bacterial communities. Proc Natl Acad Sci U S A 107:64776481. 10.1073/pnas.1000162107

Hayashi, S., Gesing, S., Quick, R., Teige, S., Ganote, C., Wu, L., and Prout, E. 2014 Galaxy based BLAST submission to distributed national high throughput computing resources. Proceedings of Science, PoS(ISGC2014)025

Juve G, Deelman E, Vahi K, Mehta G, Berriman B, Berman BP, and Maechling P. 2009. Scientific workflow applications on Amazon EC2. E-Science Workshops, 2009 5th IEEE International Conference on: IEEE. p 59-66.

Langmead B, Trapnell C, Pop M, and Salzberg SL. 2009. Ultrafast and memory-efficient alignment of short DNA sequences to the human genome. Genome Biol 10:R25. $10.1186 / \mathrm{gb}-2009-10-3-\mathrm{r} 25$

Li H, and Durbin R. 2009. Fast and accurate short read alignment with Burrows-Wheeler transform. Bioinformatics 25:1754-1760. 10.1093/bioinformatics/btp324

Li H, Ruan J, and Durbin R. 2008. Mapping short DNA sequencing reads and calling variants using mapping quality scores. Genome Res 18:1851-1858. 10.1101/gr.078212.108

Mathog DR. 2003. Parallel BLAST on split databases. Bioinformatics 19:1865-1866.

Neuhauser N, Nagaraj N, McHardy P, Zanivan S, Scheltema R, Cox J, and Mann M. 2013. High performance computational analysis of large-scale proteome data sets to assess incremental contribution to coverage of the human genome. J Proteome Res 12:28582868. 10.1021/pr400181q

Orobitg M, Guirado F, Cores F, Llados J, and Notredame C. 2015. High Performance computing improvements on bioinformatics consistency-based multiple sequence alignment tools. Parallel Computing 42:18-34.

Pellicer S, Chen G, Chan KC, and Pan Y. 2008. Distributed sequence alignment applications for the public computing architecture. IEEE Trans Nanobioscience 7:35-43. 10.1109/TNB.2008.2000148

Pordes R, Petravick D, Kramer B, Olson D, Livny M, Roy A, Avery P, Blackburn K, Wenaus T, and Frank W. 2007. The open science grid. Journal of Physics: Conference Series: IOP Publishing. p 012057. 
427 Rhoads A, and Au KF. 2015. PacBio Sequencing and Its Applications. Genomics Proteomics

$428 \quad$ Bioinformatics 13:278-289. 10.1016/j.gpb.2015.08.002

429

Rosenbloom KR, Armstrong J, Barber GP, Casper J, Clawson H, Diekhans M, Dreszer TR, Fujita PA, Guruvadoo L, Haeussler M, Harte RA, Heitner S, Hickey G, Hinrichs AS, Hubley R, Karolchik D, Learned K, Lee BT, Li CH, Miga KH, Nguyen N, Paten B,

431

432

433

434

435

436

437

438

439

440

441

442

443

444

445

446

447

448

449

450

451

452

453

454

455

456

457 Raney BJ, Smit AF, Speir ML, Zweig AS, Haussler D, Kuhn RM, and Kent WJ. 2015. The UCSC Genome Browser database: 2015 update. Nucleic Acids Res 43:D670-681. 10.1093/nar/gku1177

Schatz MC. 2009. CloudBurst: highly sensitive read mapping with MapReduce. Bioinformatics 25:1363-1369. 10.1093/bioinformatics/btp236

Scholz MB, Lo CC, and Chain PS. 2012. Next generation sequencing and bioinformatic bottlenecks: the current state of metagenomic data analysis. Curr Opin Biotechnol 23:915. 10.1016/j.copbio.2011.11.013

Søttrup CU, and Pedersen JG. 2005. Developing distributed computing solutions combining grid computing and public computing. Departamento de Ciência da Computação da universidade de Copenhagen.

Subramaniam B, and Feng W-c. 2012. The green index: A metric for evaluating system-wide energy efficiency in hpc systems. Parallel and Distributed Processing Symposium Workshops \& PhD Forum (IPDPSW), 2012 IEEE 26th International: IEEE. p 1007-1013.

White T. 2012. Hadoop: The definitive guide: " O'Reilly Media, Inc.".

Zaharia M, Chowdhury M, Franklin MJ, Shenker S, and Stoica I. 2010. Spark: cluster computing with working sets. Proceedings of the 2nd USENIX conference on Hot topics in cloud computing. Boston, MA: USENIX Association. p 10-10.

Zhang X, Wong SE, and Lightstone FC. 2014. Toward fully automated high performance computing drug discovery: a massively parallel virtual screening pipeline for docking and molecular mechanics/generalized Born surface area rescoring to improve enrichment. $J$ Chem Inf Model 54:324-337. 10.1021/ci4005145

Zook JM, Chapman B, Wang J, Mittelman D, Hofmann O, Hide W, and Salit M. 2014. Integrating human sequence data sets provides a resource of benchmark SNP and indel genotype calls. Nat Biotechnol 32:246-251. 10.1038/nbt.2835

458 


\section{Figure 1 (on next page)}

Processing time of BLAST sequence alignment by number of reads analyzed on a single personal computer.

One, 10, 100 and 1000 reads were analyzed in triplicate. The times shown were average processing times of triplicate runs. 


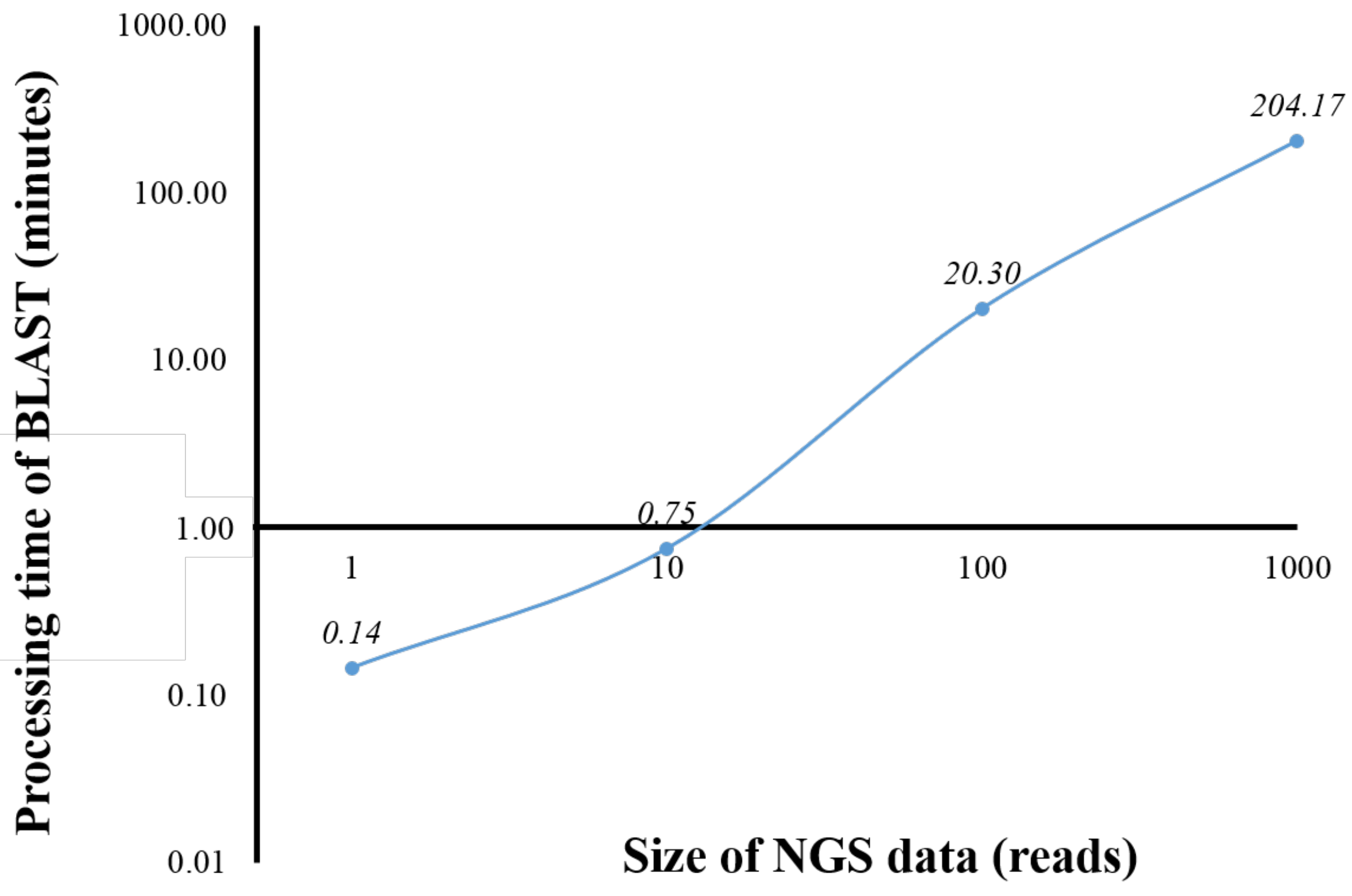




\section{Figure 2 (on next page)}

Processing time of BLAST sequence alignment by number of reads analyzed on HPC.

One, 10, 100, 1000 and 10000 reads were analyzed in triplicate. The times shown were average processing times of triplicate runs. Only one run for BLAST analysis was performed for 100000 reads due to an extensive processing time. 


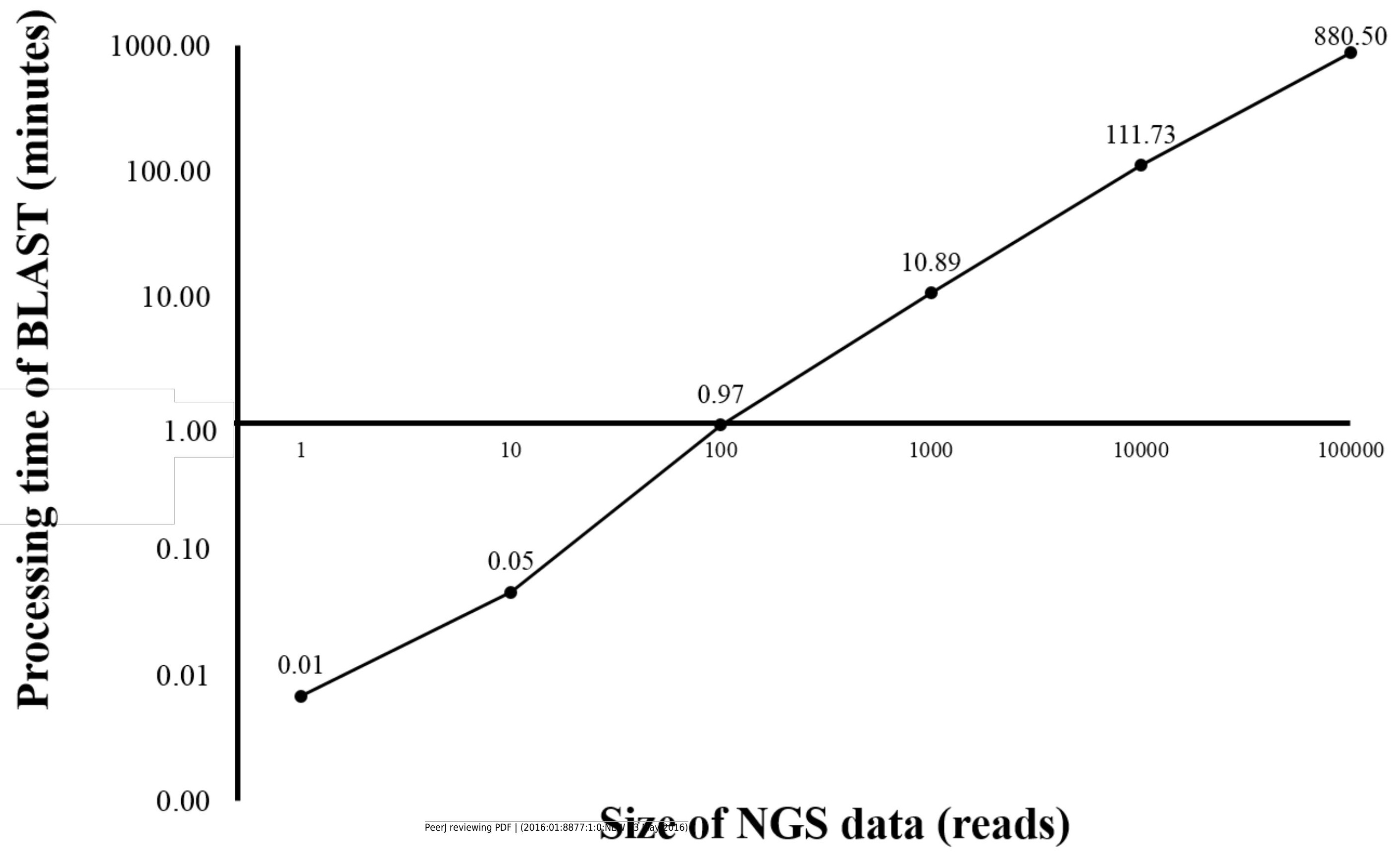




\section{Figure 3 (on next page)}

Histogram of sequence alignment times for each WU.

Five thousand WUs were distributed through a BOINC grid system with 50 client machines in one 14-hour-long session. Each WU contained 100 sequence reads of the total 500000 reads. 


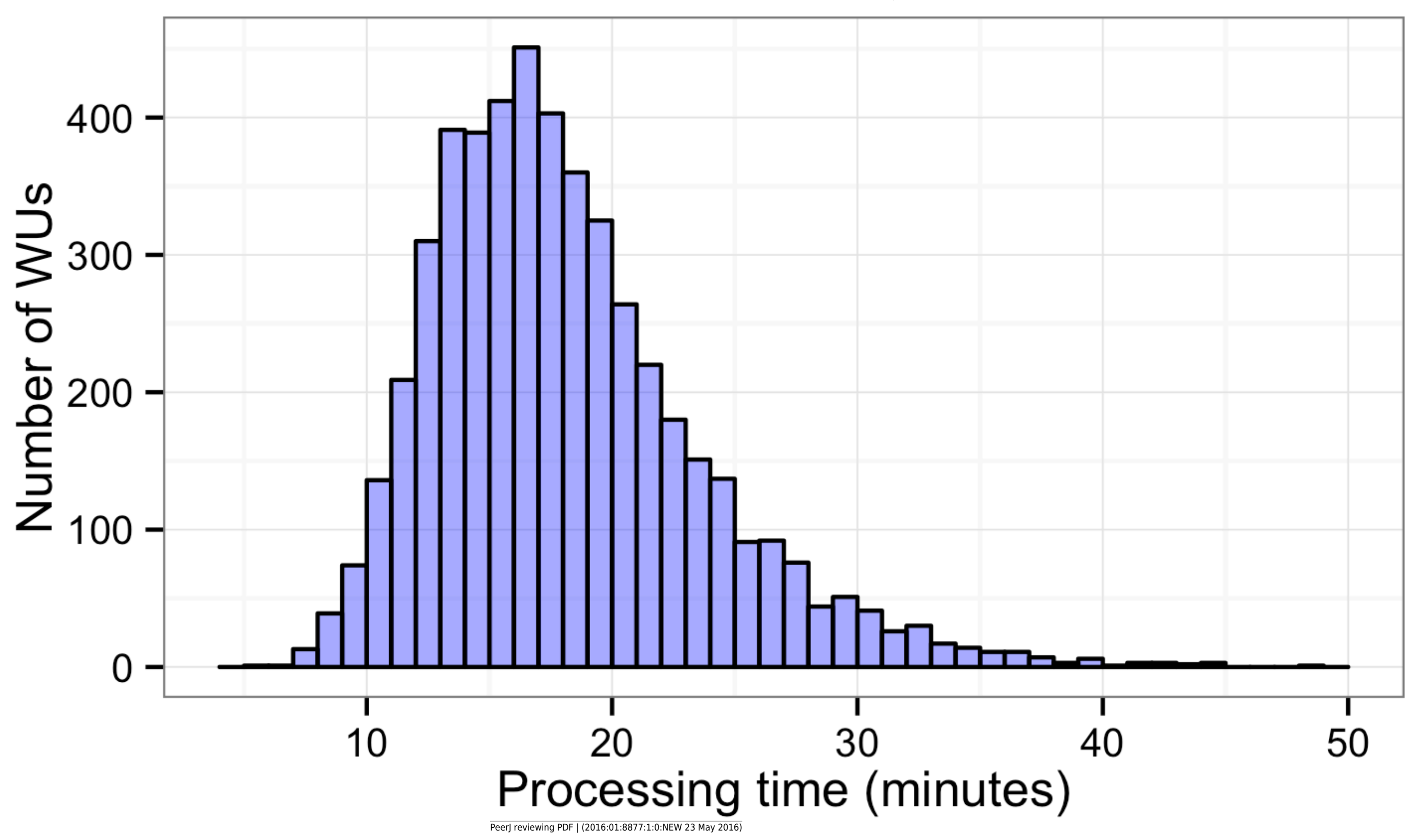


Figure 4(on next page)

Histograms of the performance of client machines during one 14-hour-long session of BLAST analysis through BOINC grid system.

Average processing time per WU of each client machine was measured (A). Time used for data transfer of each WU was recorded (B). Finally, the total number of WUs each client machine handled during the sessions was recorded (C). 


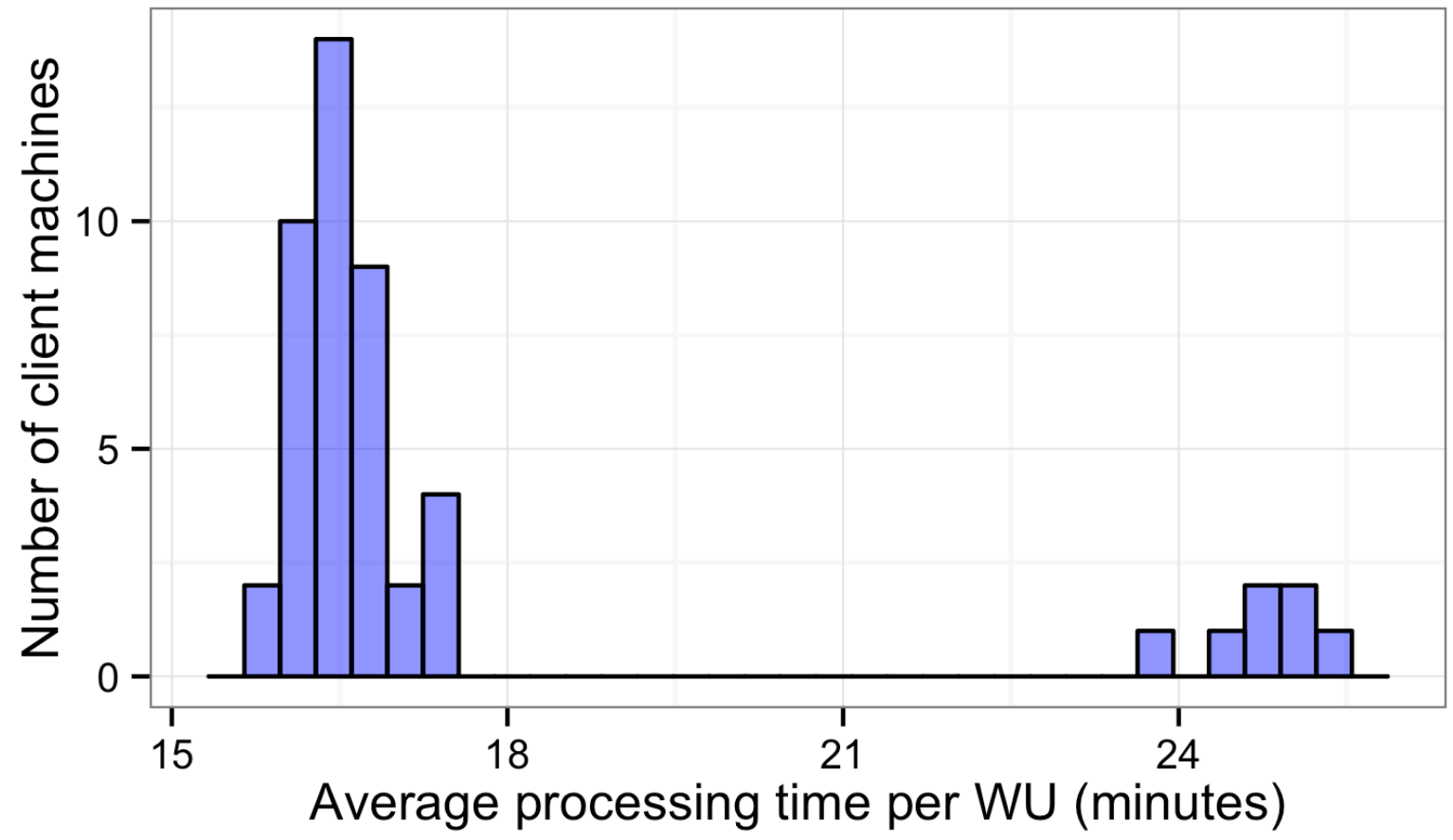

B

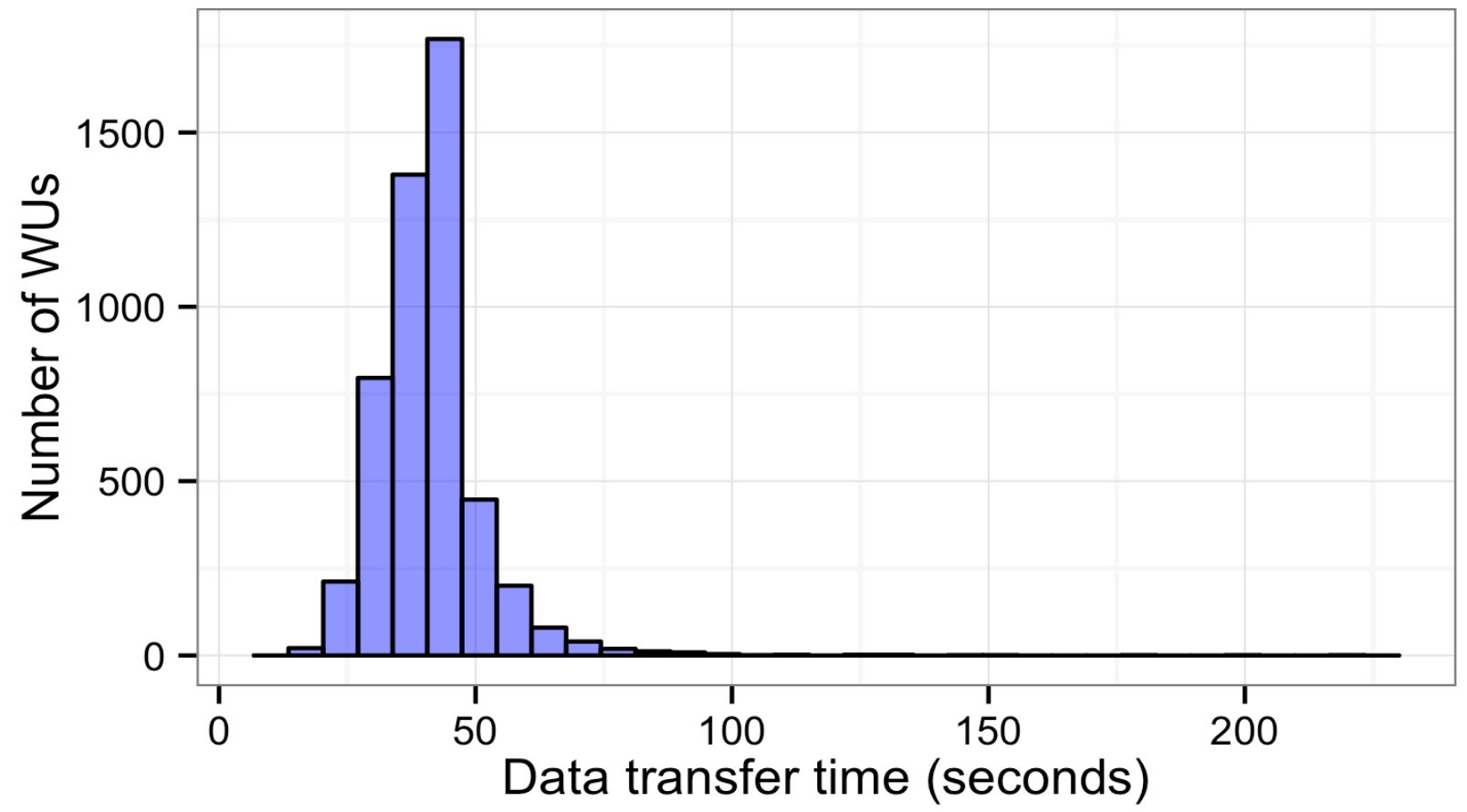

C

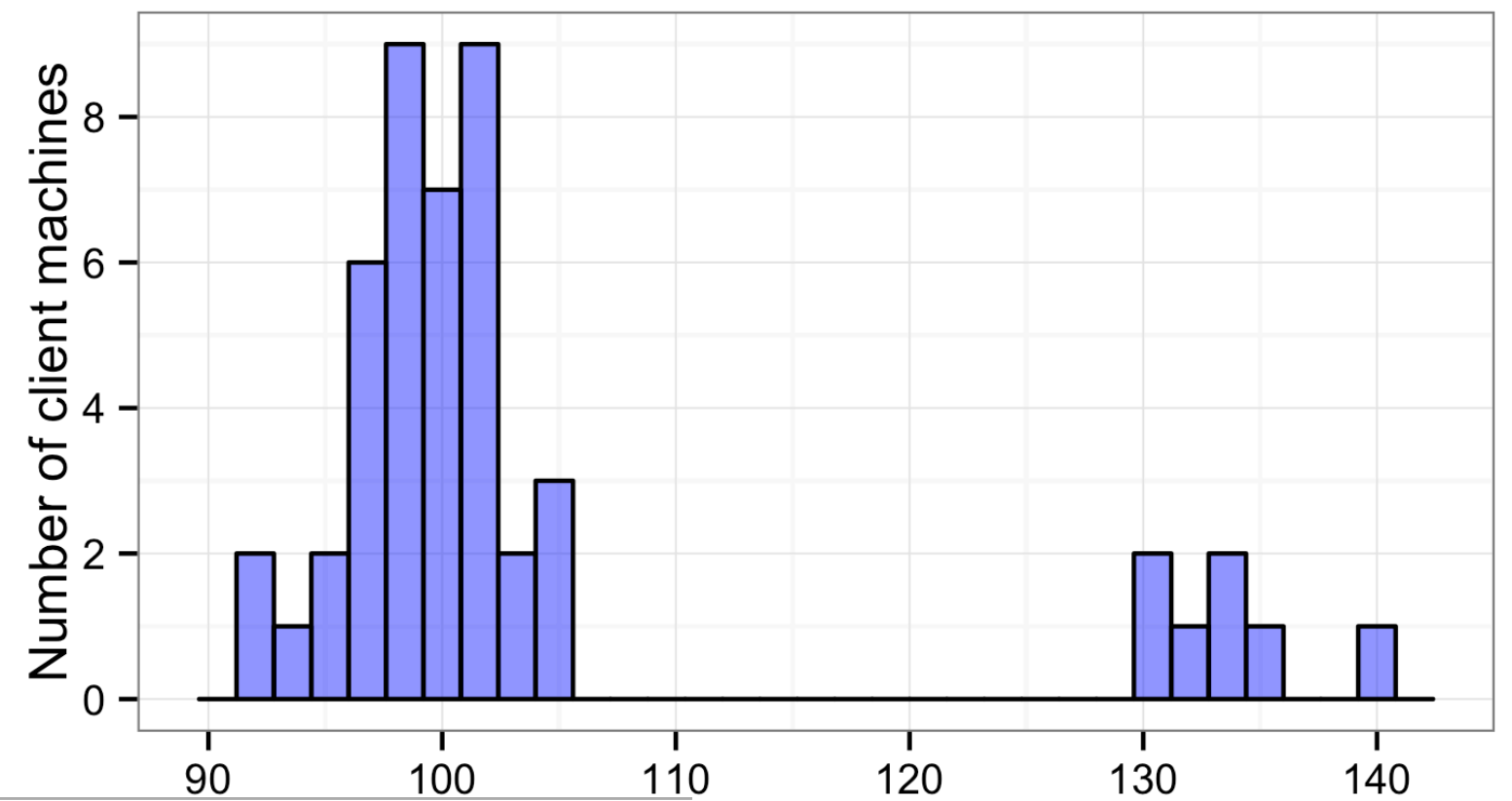

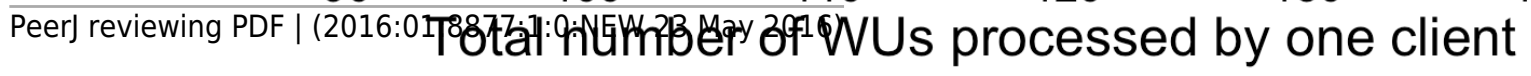




\section{Figure 5 (on next page)}

Number of WUs under processing at a particular time.

Two machines with the longest (Slow, blue line) and the shortest (Fast, red line) average processing time per WU were tracked for the number of WUs under processing in parallel during the overnight session. Small peaks happened as the transfer of completed WUs and new WUs were overlapping. 


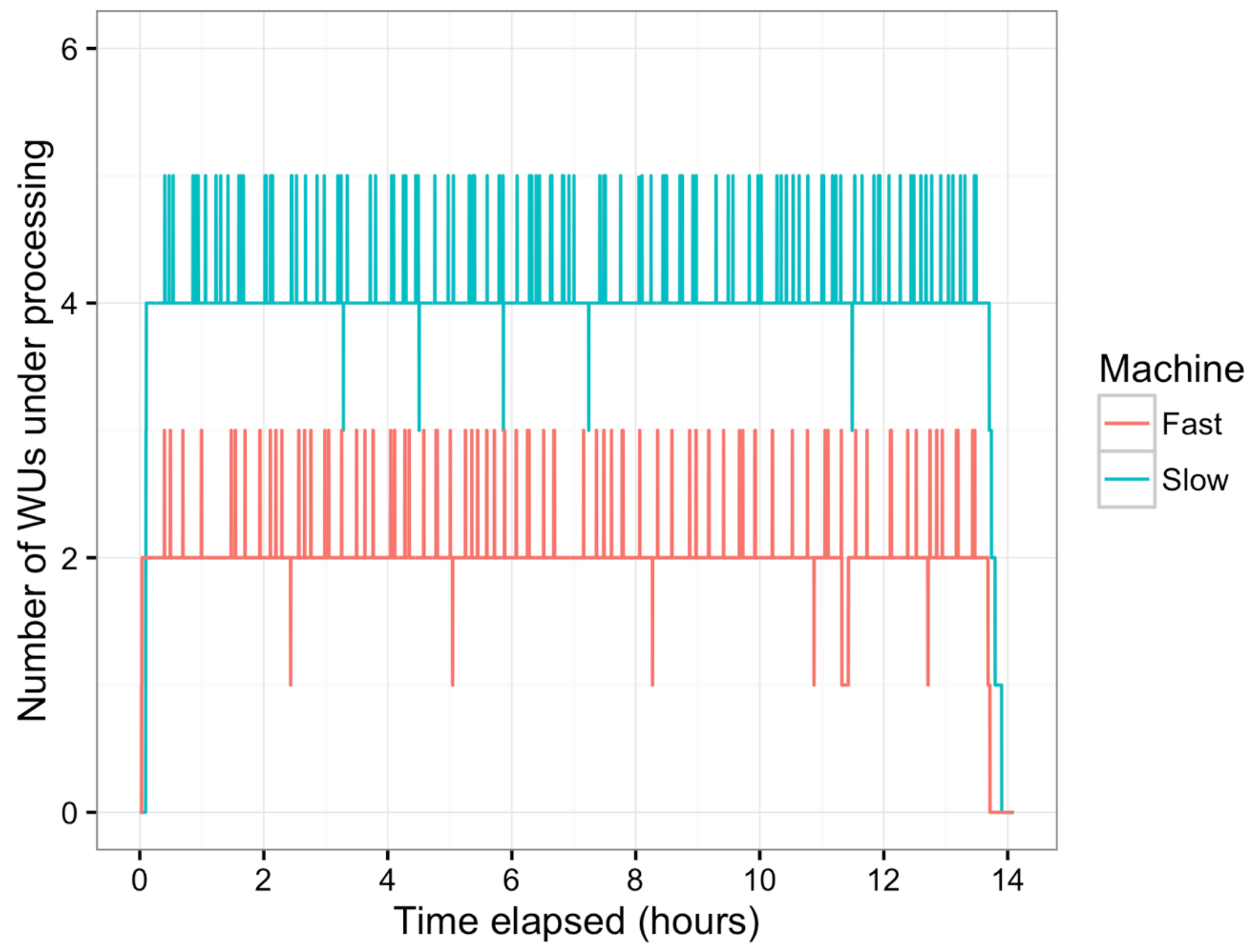

